\title{
The "CHROME criteria": Tool to optimize and audit prescription quality of psychotropic medications in institutionalized people with dementia
}

\author{
Ruben Muñiz, ${ }^{1}$ Alia I. Pérez-Wehbe, ${ }^{2}$ Francisco Couto, ${ }^{3}$ María Pérez, ${ }^{3}$ Noemí Ramírez, ${ }^{3}$ \\ Alejandro López, ${ }^{3}$ Javier Rodríguez, ${ }^{3}$ Teresa Usieto, ${ }^{3}$ Lietzan Lavin, ${ }^{3}$ Ana Rigueira, ${ }^{4}$ \\ Luis Agüera-Ortiz, ${ }^{5}$ Jorge López-Alvarez, ${ }^{5}$ Manuel Martín-Carrasco, ${ }^{6}$ \\ and Javier Olazarán $n^{1,7}$ \\ ${ }^{1}$ Maria Wolff Foundation, Madrid, Spain \\ ${ }^{2}$ Universidad de la Laguna, Sta Cruz de Tenerife, Spain \\ ${ }^{3}$ Centro Sociosanitario El Pino, Instituto de Atención Social y Sociosanitaria (IAS), Las Palmas de Gran Canaria, Spain \\ ${ }^{4}$ Servicio de Farmacia, Hospital Universitario San Agustín, Avilés, Spain \\ ${ }^{5}$ Servicio de Psiquiatría, Hospital Universitario 12 de Octubre, Madrid, Spain \\ ${ }^{6}$ Clinica Padre Menni, Hermanas Hospitalarias, Pamplona, Spain \\ ${ }^{7}$ Servicio de Neurologia, Hospital General Universitario Gregorio Marañón, Madrid, Spain
}

Objective: Describe and validate the CHROME (CHemical Restraints avOidance MEthodology) criteria.

Design: Observational prospective longitudinal study.

Setting: Single nursing home in Las Palmas de Gran Canaria, Spain.

Participants: 288 residents; mean age: 81.6 (SD 10.6). $77.4 \%$ had dementia.

Intervention: Multicomponent training and consultancy program to eliminate physical and chemical restraints and promote overall quality care. Clinicians were trained in stringent diagnostic criteria of neuropsychiatric syndromes and adequate psychotropic prescription.

Measurements: Psychotropic prescription (primary study target), neuropsychiatric syndromes, physical restraints, falls, and emergency room visits were semi-annually collected from December 2015 to December 2017. Results are presented for all residents and for those who had dementia and participated in the five study waves (completer analysis, $n=107$ ).

Results: For the study completers, atypical neuroleptic prescription dropped from $42.7 \%$ to $18.7 \%$, long half-life benzodiazepines dropped from $25.2 \%$ to $6.5 \%$, and hypnotic medications from $47.7 \%$ to $12.1 \%(p<0.0005)$. Any kind of fall evolved from 67.3 to 32.7 (number of falls by 100 residents per year). Physicians' diagnostic confidence increased, while the frequency of diagnoses of neuropsychiatric syndromes decreased $(p<0.0005)$.

Conclusions: Implementing the CHROME criteria reduced the prescription of the most dangerous medications in institutionalized people with dementia. Two independent audits found no physical or chemical restraint and confirmed prescription quality of psychotropic drugs. Adequate diagnosis and independent audits appear to be the keys to help and motivate professionals to optimize and reduce the use of psychotropic medication. The CHROME criteria unify, in a single compendium, neuropsychiatric diagnostic criteria, prescription guidelines, independent audit methodology, and minimum legal standards. These criteria can be easily adapted to other countries.

Key words: Chemical restraint, Nursing home, Pharmacological restraint, Pharmacological treatment, Neuropsychiatric symptoms, Dementia, Psychotropic medication, Non-pharmacological therapies

Correspondence should be addressed to: Javier Olazarán, MD, PhD, Fundación Maria Wolff, Río Sil 15 bis, 28660 Boadilla del Monte, Madrid, Spain. Phone: +34 916322507; Fax +34 914336033. Email: javier@mariawolff.es. Received 04 Feb 2019; revision requested 22 Mar 2019; revised version received 07 Jun 2019; accepted 19 Jun 2019. First published online 22 October 2019.

\section{Introduction}

Despite their modest efficacy and the significant risks derived from their use, psychotropic medications are widely utilized among elderly adults with cognitive 
impairment, often in a nonspecific way (Majic et al., 2010). Neuroleptics and long half-life benzodiazepines are the most deleterious drugs associated with faster cognitive decline, higher rate of cerebrovascular events and falls, and unexpected high mortality (Ballard et al., 2009; Olazarán et al., 2013; Schneider et al., 2005). The term chemical restraint has been created to designate the inappropriate prescription of drugs that have the ability to limit personal freedom.

Namely, the Centers for Medicare \& Medicaid Services (CMS) and the federal law of the United States defined chemical restraint as "any drug used for discipline or convenience and not required to treat medical symptoms." The CMS defines "convenience" (Interpretive Guidelines $\$ 483.13(\mathrm{a})$ ) as any action taken by the facility to control a resident's behavior with a lesser amount of effort by the facility and not in the resident's best interest. "Medical symptom" is defined as an indication or characteristic of a physical or psychological condition.

Although these definitions and interpretations help identify extreme cases of malpractice, less obvious cases of pharmacological restraining or suboptimal prescription quality might pass undetected as concepts like "resident's best interest" or "physical or psychological condition" are vague and prone to biased interpretation. This is possibly one of the reasons why there is little research on chemical restraints as such, and most studies approach the subject via prevalence of psychotropic medication prescription with focus on antipsychotics, anxiolytics, and hypnotics (Hughes and Lapane, 2005; Phillips et al., 2000; Richter et al., 2012).

To reduce the use of chemical restraint and promote quality prescription of psychotropic drugs in institutionalized settings, a panel of experts created the CHROME (CHemical Restraints avOidance MEthodology) criteria (Olazarán-Rodríguez et al., 2016). The panel members were experts from psychiatry, neurology, geriatrics, psychology, pharmacology, as well as the legal and managerial fields. The organizational and procedural aspects of the CHROME criteria were designed for institutionalized people with dementia. However, the diagnostic and treatment recommendations allow application in other situations where disruptive disorders may potentially put the person at risk of being subjected to chemical restraint.

The CHROME criteria define chemical restraint as: a) any drug prescribed out of organizational convenience and/or b) any drug that is not prescribed to treat any of the six neuropsychiatric syndromes (as defined in Appendix Table 1) that cause significant patient suffering. In contrast to the definition of the CMS, this more precise definition allows an external physician to more accurately identify possible or definite chemical restraint as well as making quality prescription audits feasible. Furthermore, these criteria provide listings of admissible drugs and dosages for each syndrome; norms for the acquisition, use, and disposal of psychotropic drugs; legal requirements like the kind of informed consent by type of situation; and most importantly, a method to externally verify (audit) the prescription quality of psychotropic drugs of any given nursing home. In the case of a favorable audit result, the home is awarded an accreditation of "Chemical restraint-free home according to the CHROME criteria," backed by the Confederación Española de Asociaciones de Familiares de Enfermos de Alzheimer (CEAFA) i.e. the Spanish National Alzheimer's Association. An updated version of the CHROME criteria is freely available as an online appendix to this article.

The objective of this validation study was to describe the evolution of psychotropic drug prescription as well as other potentially related variables during the training and consultancy process. The several components involved culminated with the accreditation of a nursing home as a "chemical restraint-free center," issued by CEAFA, which independently made the audit applying Maria Wolff Foundation's CHROME criteria. On November 2017, the Association's chairperson issued the accreditation confirming that the facility fully complied with all the requirements set by the CHROME criteria. Neither definitive nor possible chemical restraints were found, and quality prescription criteria were met on randomly selected cases as well as those cases picked by the auditor after visual inspection of the home (Appendix Table 1). The figures of the study's last wave were recorded two weeks after the audit. In addition to drug-related figures, the study reports the evolution of fall rates, physical restraints, and diagnoses of neuropsychiatric syndromes.

\section{Methods}

\section{Design}

This was an observational, longitudinal, prospective study in which data of residents of a single nursing home were semi-annually extracted at six dates (study waves) from December 2015 to December 2017 .

\section{Ethics}

Approval for the study was obtained from an institutional ethics committee (University of La Laguna, Tenerife, Spain). 


\section{Setting}

The nursing home that was object of the present study is integrated in a lager sociosanitary complex located in the city of Las Palmas on the island of Gran Canaria (Spain). Five wards with 215 beds for people with dementia or highly dependent elderly people were included in the study. The other four wards offer day care for the elderly with or without dementia and 72 mental health beds. A total of 448 people are cared for. The "Centro Sociosanitario El Pino" has been managed since 2010 by the ICOT Health Group through a contract with the local authority "Instituto de Atención Social y Sociosanitaria" of the "Cabildo de Gran Canaria." Led by the Maria Wolff Foundation, El Pino initiated in 2014 a comprehensive training program for all its professionals aimed at preventing and treating behavioral and psychological symptoms of dementia (BPSD), eliminating physical and chemical restraints, and promoting dementia-friendly care and person-centered care to all its residents. Since the program was conceived and developed for the care of institutionalized people with dementia, the psychiatric and day care residents were not included in the study.

It is noteworthy that El Pino holds unusually good staffing ratios for Spanish standards. For example, nurse aid staffing is more than adequate (e.g. two nurse aids in the night shift per ward of 40 residents, in addition to other medical staff like registered nurses or 24-hour medical doctor). In addition, four full-time psychologists and five medical doctors under one medical coordinator provide psychological and medical care for the 215 residents. Activities manager, occupational therapists, and physiotherapists are also available with an adequate staff to resident ratio. Senior and middle management positions are held in part by psychologists, which contributes to the organization's commitment with nonpharmacologic treatment and psychoactive drug treatment containment.

\section{Study variables}

The primary outcome variable was the frequency of prescription of psychotropic medications, which were divided into the following categories: typical neuroleptics, atypical neuroleptics, antidepressants, hypnotics (short/intermediate half-life benzodiazepines, benzodiazepine derivatives, clomethiazole), long half-life benzodiazepines, dementia-specific medications (cholinesterase inhibitors [CEI], memantine), and antiepileptics. The prescription of psychotropics was coded as yes or not, either continuous or PRN prescription, in the previous week to the study wave. In the case of continuous prescriptions, the daily dose was also registered.
In addition, the following study variables were collected:

- Age and sex, in the first date of study inclusion.

- Total number of prescribed medications for any condition, as registered in the electronic medical history, at the date of the study wave.

- Dementia, when the resident was first included in the study, according to American Psychiatric Association criteria (2013) or $<24$ score in the Spanish version of the Mini-Mental State Examination (Lobo et al., 1999).

- Presence of neurospychiatric syndromes, according to CHROME criteria (Appendix Table 1), at the date of the study waves, as diagnosed by the consensus of psychologist and physician. The category of possible syndrome was utilized when there was suspicion of neuropsychiatric syndrome without sufficient diagnostic confidence or information.

- Performance in basic activities of daily living, according to the Barthel Index (Cid-Ruzafa and Damián-Moreno, 1997), closest to the study waves.

- Risk of falling, according to the Downton Index (Aranda-Gallardo et al., 2015), closest to the study waves.

- Falls accumulated in the six months before the date of the study wave. Three types of falls were recorded: non-injurious falls, falls with hip fracture, and falls with any other complication.

- Any kind of physical restraint for at least a week, at the date of the study wave. Bed rails were excluded from the definition of physical restraint in accordance with previous reports (Ljunggren et al., 1997).

- Referrals to the emergency room department in the six months previous to the date of the study waves.

- Death, when occurred between two consecutive study waves.

Since the specific CHROME training was initiated on April 2016 (i.e. between the dates of the first and second study waves), the diagnoses of neuropsychiatric syndromes were not collected at the first study wave. Falls and emergency room referrals began to be systematically recorded on December 1, 2015, and, for that reason, data regarding those variables were also lacked for the first wave. All the other study variables were prospectively collected as part of the center's usual assessment protocol. Figures of falls, emergency room referrals, and death were expressed as number of events per 100 fully occupied beds per year (RPY).

\section{Intervention}

A total of four training sessions adding up to 16 hours per employee were delivered to about 200 staff members over a period of three years. The employees who were trained ranged from senior management and physicians to nurse aids or maintenance personnel. These programs had the transformative 


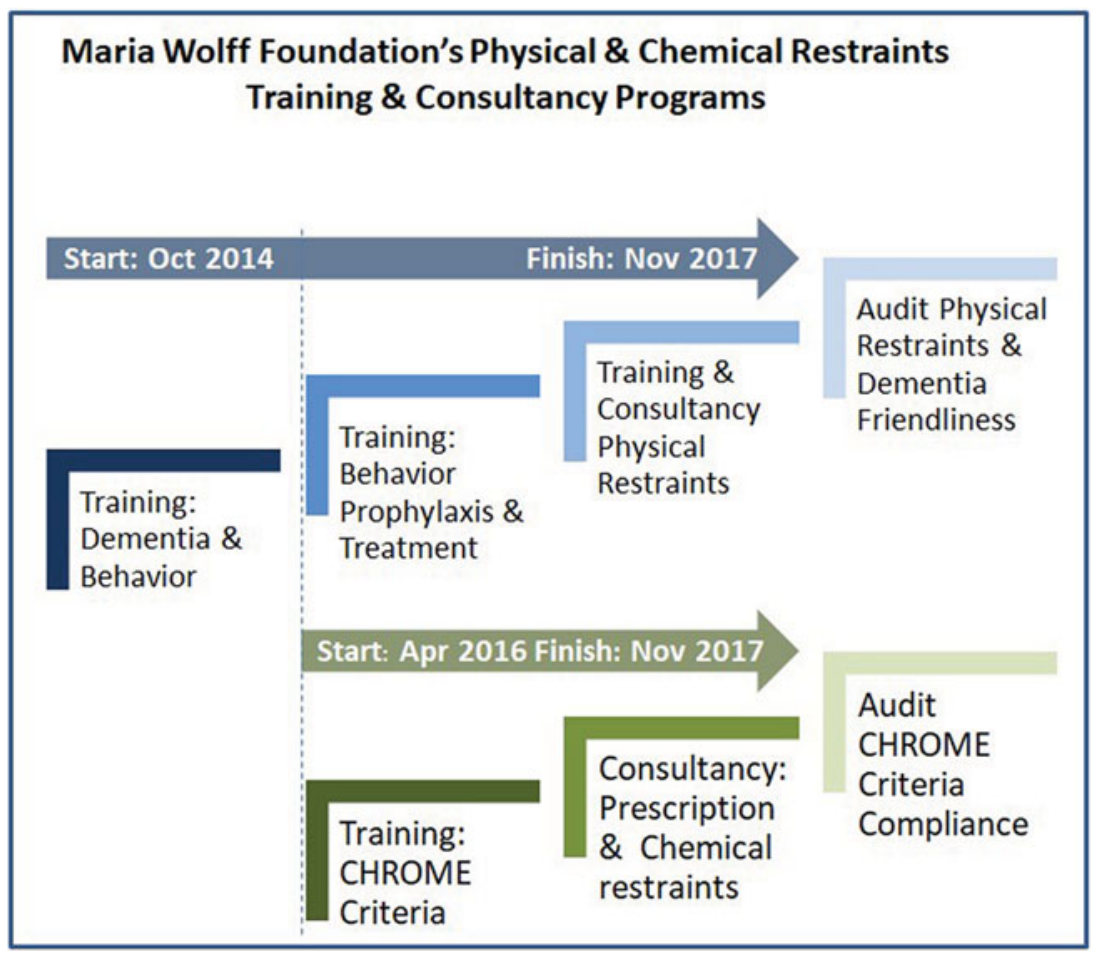

Figure 1. Maria Wolff Foundation's physical and chemical restraints training and consultancy programs.

aims to a) build a dementia friendly culture, b) prevent and treat BPSD non-pharmacologically or in combination with drugs, and c) implement a comprehensive physical restraint reduction program. Implementation of the three programs was systematically followed up with formal videoconferences covering consultancy and discussion of clinical cases. The essential components of the Maria Wolff Foundation physical restraint-free care program for nursing homes have been described in detail elsewhere (Muñiz et al., 2016). These components are basically training, consultation, and consultancy provided at various levels of the organization with special focus on genesis, prophylaxis and management of BPSD, environmental modifications, and person-centered care philosophy. The steps and contents of the intervention implemented in El Pino are summarized in Figure 1.

Once the physical restraint reduction program was fully under way, the CHROME criteria were implemented simultaneously: medical doctors, psychologists, nurses, managers, and the pharmacist received eight hours of training in CHROME criteria. To identify possible or definitive chemical restraints or inappropriate prescription, all the patients had to be diagnosed at each study wave. To ensure diagnostic accuracy, psychologists and medical doctors made their diagnoses separately and tried to reach diagnostic consensus case by case. These diagnoses were the basis by which physicians then applied the prescription guidelines delivered by the CHROME criteria. The center's pharmacist helped to avoid undesired interactions and kept the study database up to date regarding drugs. The study director of the CHROME criteria (JO) provided ad libitum videoconference, e-mail, and telephone support.

\section{Data extraction and statistical analysis}

Pharmacological and medical data were extracted from the center's software/database (Aegerus, Sabadell, Spain) to MS Excel 2010 (Microsoft, Redmond, WA) by the center investigators. Diagnostic data were produced by the medical doctors and psychologists and manually recorded in the mentioned spreadsheet. Data were reviewed for potential artifacts and errors by the principal investigator $(\mathrm{RM})$, the study director (JO), and one of the center's psychologists (FC) and then exported from MS Excel into SPSS v.15.0 (SPSS Inc., Chicago, IL) for analysis.

Measures of frequency, central tendency, and dispersion were utilized to describe the residents' characteristics and to analyze the evolution of the study variables across the five study waves. The evolution of residents who had dementia and remained in the nursing home throughout the complete study period was specifically analyzed (completer analysis) as these were the cases that can benefit most from the study intervention and therefore validate the CHROME criteria. Nonparametric statistics 
Table 1. Study variables in the total sample of residents across the five study waves

\begin{tabular}{|c|c|c|c|c|c|}
\hline & $\begin{array}{c}12 / 01 / 2015 \\
(n=205)\end{array}$ & $\begin{array}{c}06 / 01 / 2016 \\
(n=217)\end{array}$ & $\begin{array}{c}12 / 01 / 2016 \\
(n=214)\end{array}$ & $\begin{array}{c}06 / 01 / 2017 \\
(n=219)\end{array}$ & $\begin{array}{c}12 / 01 / 2017 \\
(n=214)\end{array}$ \\
\hline Age & $82.7(10.1)$ & $82.5(10.6)$ & $81.9(10.5)$ & $81.6(10.6)$ & $81.3(10.7)$ \\
\hline Sex ( $\%$ female $)$ & 66.3 & 64.8 & 62.6 & 62.4 & 61.6 \\
\hline Basic ADL (BI) & $22.2(26.6)$ & $25.0(28.3)$ & $23.8(28.0)$ & $25.1(27.4)$ & $24.3(27.5)$ \\
\hline Physical restraint (\%) & $35.1(28.6-41.7)$ & $10.6(6.5-14.7)$ & $7.5(4.0-11.0)$ & $6.4(3.2-9.6)$ & 0 \\
\hline Risk of falling (DI) & $4.5(1.5)$ & $4.5(1.5)$ & $4.4(1.5)$ & $4.4(1.5)$ & $4.5(1.5)$ \\
\hline Fall of any type (RPY) & NC & 100.7 & 136.6 & 90.0 & 135.4 \\
\hline Noninjurious falls & $\mathrm{NC}$ & 88.7 & 118.6 & 68.2 & 105.1 \\
\hline Falls with hip fracture & $\mathrm{NC}$ & 0.00 & 1.99 & 0.95 & 0.00 \\
\hline Falls other complications & NC & 12.0 & 15.9 & 20.8 & 30.3 \\
\hline $\begin{array}{l}\text { Emergency room referral } \\
\text { (RPY) }\end{array}$ & NC & 35.9 & 78.7 & 49.2 & 44.5 \\
\hline Death (RPY) & 29.4 & 20.9 & 18.9 & 17.0 & 16.1 \\
\hline Medications (n) 1 & $8.2(3.2)$ & $7.1(3.3)$ & $6.9(3.1)$ & $7.1(3.2)$ & $6.4(3.4)$ \\
\hline Typical neuroleptics (\%) & $7.5(3.8-11.1)$ & $5.6(2.5-8.7)$ & $5.7(2.5-8.8)$ & $5.1(2.2-8.1)$ & $3.8(1.2-6.5)$ \\
\hline Atypical neuroleptics (\%) & $43.8(36.9-50.6)$ & $36.0(29.6-42.4)$ & $37.4(30.9-43.9)$ & $38.2(31.8-44.7)$ & $27.8(21.7-33.7)$ \\
\hline Antidepressants (\%) & $45.0(38.1-51.9)$ & $38.2(32.1-45.2)$ & $40.2(34.1-47.2)$ & $43.3(37.2-50.4)$ & $41.6(35.4-48.7)$ \\
\hline Hypnotics (\%) & $35.6(29.1-42.2)$ & $34.7(28.4-41.0)$ & $34.1(27.8-40.5)$ & $30.1(24.2-36.1)$ & $16.4(11.5-21.3)$ \\
\hline Long half-life BZD (\%) & $24.4(18.5-30.3)$ & $13.2(8.8-17.7)$ & $14.5(9.8-19.2)$ & $13.5(9.1-18.0)$ & $9.6(5.7-13.5)$ \\
\hline $\mathrm{CEI} / \mathrm{memantine}(\%)$ & $38.0(31.3-44.7)$ & $35.4(28.9-41.8)$ & $36.0(29.6-42.4)$ & $36.9(30.4-43.3)$ & $35.5(29.1-41.9)$ \\
\hline Antiepileptics (\%) & $18.4(13.1-23.8)$ & $17.3(12.2-22.4)$ & $17.9(12.8-23.1)$ & $19.8(14.5-25.1)$ & $19.2(13.9-24.4)$ \\
\hline
\end{tabular}

Figures represent mean (SD), frequency (95\% confidence interval), or number of events per 100 beds per year (RPY); ${ }^{1} \mathrm{PRN}$ prescriptions are included. ADL: activities of daily living; BI: Barthel Index; BZD: benzodiazepines; CEI: cholinesterase inhibitors; DI: Downton Index; n: number; NC: not collected.

were applied for the analysis of psychotropic medication prescription and other study variables in those subjects, taking as reference the first measurement. Given that frequency of psychotropic prescription was the primary study outcome and since there were seven groups of psychotropic medications, the level of statistical significance was set at $\mathrm{p}<0.007$.

\section{Results}

A total of 288 residents were studied across the five study waves. Mean age at study inclusion was 81.6 years (SD 10.6, range 38-104), $183(63.5 \%)$ of the participants were female and $223(77.4 \%)$ of them had dementia. The demographic and clinical variables of the residents across the five study waves are presented in Table 1. As expected, the participants were polymedicated, had a high risk of falls, and had high functional dependence. There was a prevalence of physical restraint of $35.1 \%$ in the first wave, which was progressively reduced and culminated with complete absence of physical restraints at the end of the study. Those results were accompanied by an increase of noninjurious falls (from 88.7 to 105.1 RPY), falls with complication different from hip fracture (from 12.0 to $30.3 \mathrm{RPY}$ ), and emergency room referrals (from 35.9 to $44.5 \mathrm{RPY}$ ). However, falls with hip fracture did not increase significantly, and demise was progressively decreased across the study waves (Table 1 ).

The mean number of psychotropic prescriptions per resident was 1.6 (SD 1.3 , range $0-5$ ) at the beginning of the study and 1.0 (SD 1.1, range 0-5) at the end. At the beginning of the study, prescription was particularly frequent for antidepressants $(45.0 \%)$, atypical neuroleptics (43.8\%), and hypnotics $(35.6 \%)$. At the end of the study, frequency of prescription was significantly reduced for atypical neuroleptics, long half-life benzodiazepines, and hypnotic medications (Table 1). "As needed" (PRN) prescriptions were frequently observed for neuroleptics, long half-life benzodiazepines, or hypnotics at the beginning of the study (30.7\% [CI 95\% 22.4-37.0]) but were reduced at the end (4.7\% [CI 95\% 1.8-7.5]). Significant dose changes could not be detected across the study waves, although the number of patients that remained on the different medications was low. For instance, the mean dose (SD) of quetiapine the most frequently prescribed neuroleptic- was $140.6(115.7) \mathrm{mg} / \mathrm{d}$ in the first wave $(n=28)$ and $132.1(77.5) \mathrm{mg} / \mathrm{d}$ in the last wave $(n=14)$; the corresponding figures for lormetazepam, which was the most frequently prescribed hypnotic, were $1.4(0.9) \mathrm{mg} / \mathrm{d}(n=12)$ and $0.9(0.2) \mathrm{mg} / \mathrm{d}$ $(n=7)$ (all $\mathrm{p}$ values $>0.05$, results of medication dose are not shown). 
Table 2. Results in the residents which had dementia and completed the five study waves $(n=107)$

\begin{tabular}{|c|c|c|c|c|c|c|}
\hline & $12 / 01 / 2015$ & $06 / 01 / 2016$ & $12 / 01 / 2016$ & $06 / 01 / 2017$ & $12 / 01 / 2017$ & $p^{2}$ \\
\hline Basic ADL (BI) & $19.2(23.8)$ & $20.8(24.2)$ & $17.8(23.1)$ & $17.3(21.9)$ & $16.8(21.7)$ & 0.021 \\
\hline Physical restraint (\%) & $43.0(33.6-52.4)$ & $15.9(9.0-22.8)$ & $12.1(6.0-18.3)$ & $11.2(5.2-17.2)$ & 0 & 0.000 \\
\hline Risk of falling (DI) & $4.6(1.4)$ & $4.6(1.5)$ & $4.3(1.6)$ & $4.0(1.7)$ & $3.6(1.7)$ & 0.000 \\
\hline Fall, any type (RPY) & $\mathrm{NC}$ & 67.3 & 72.0 & 35.5 & 32.7 & NA \\
\hline Non-injurious fall & $\mathrm{NC}$ & 59.8 & 68.2 & 27.1 & 23.4 & NA \\
\hline Falls with hip fracture & $\mathrm{NC}$ & 0 & 0.9 & 0 & 0 & NA \\
\hline $\begin{array}{l}\text { Falls with other } \\
\text { complication }\end{array}$ & NC & 7.5 & 2.8 & 8.4 & 9.3 & NA \\
\hline $\begin{array}{c}\text { Emergency room } \\
\text { referral (RPY) }\end{array}$ & 20.6 & 12.1 & 34.6 & 9.3 & 7.5 & NA \\
\hline Medications (n) ${ }^{1}$ & $8.3(3.5)$ & $6.7(3.0)$ & $6.6(3.2)$ & $6.5(2.9)$ & $5.6(2.8)$ & 0.000 \\
\hline Typical neuroleptics & $5.8(1.3-10.3)$ & $2.9(0.0-6.1)$ & $2.8(0.0-6.0)$ & $1.9(0.0-4.5)$ & $1.9(0.0-4.5)$ & 0.102 \\
\hline Atypical neuroleptics & $42.7(33.2-52.3)$ & $33.3(24.3-42.4)$ & $34.6(25.6-43.6)$ & $34.6(25.6-43.6)$ & $18.7(11.3-26.1)$ & 0.000 \\
\hline Antidepressants & $45.6(36.0-55.3)$ & $40.0(30.6-49.4)$ & $43.0(33.6-52.4)$ & $42.1(32.7-51.4)$ & $40.2(30.9-49.5)$ & 0.257 \\
\hline Hypnotics & $47.7(38.2-57.1)$ & $40.2(30.9-49.5)$ & $38.3(29.1-47.5)$ & $38.3(29.1-47.5)$ & $12.1(6.0-18.3)$ & 0.000 \\
\hline $\begin{array}{l}\text { Long half-life BZD } \\
(\%)\end{array}$ & $25.2(17.0-33.5)$ & $11.2(5.2-17.2)$ & $15.0(8.2-21.7)$ & $14.0(7.4-20.6)$ & $6.5(1.9-11.2)$ & 0.000 \\
\hline $\mathrm{CEI} /$ memantine & $53.4(43.8-63.0)$ & $48.6(39.0-58.1)$ & $47.7(38.2-57.1)$ & $45.8(36.4-55.2)$ & $46.7(37.3-56.2)$ & 0.059 \\
\hline Antiepileptics & $15.5(8.5-22.5)$ & $14.3(7.6-21.0)$ & $15.0(8.2-21.7)$ & $15.0(8.2-21.7)$ & $14.0(7.4-20.6)$ & 0.739 \\
\hline
\end{tabular}

Figures represent mean (SD), frequency (95\% confidence interval), or number of events per 100 beds per year (RPY).

${ }^{1}$ PRN prescriptions are included; ${ }^{2}$ for the comparison between the last and the first measurement (Wilcoxon test). ADL: activities of daily living; BI: Barthel Index; BZD: benzodiazepines; CEI: cholinesterase inhibitors; DI: Downton Index; n: number; NA: not applicable; NC: not collected; RPY: per 100 beds and year.

There were 107 residents with dementia, who remained in the nursing home during the complete study period. The mean (SD) age of those residents was 84.2 (8.5) years, and $76.6 \%$ of them were female. The evolution of the study variables of these completer subjects is presented in Table 2. In this group, a significant reduction of psychotropic medication was confirmed for atypical neuroleptics, which fell from $42.7 \%$ to $18.7 \%$ of prescription frequency; for hypnotics, which fell from $47.7 \%$ to $12.1 \%$, and for long half-life benzodiazepines, which fell from $25.2 \%$ to $6.5 \%$. Considering only neuroleptic, long half-life benzodiazepines, and hypnotic medications, the total number of prescriptions per patient fell from 1.1 (SD 1.1 , range $0-5$ ) to 0.4 (SD 0.7, range $0-3$ ), $\mathrm{p}<0.05$ (Figure 2). The observed reduction of psychotropic prescription and elimination of physical restraints were accompanied by a decrease of falls (from 67.3 RPY to $32.7 \mathrm{RPY}$ ) and a decrease of emergency room referrals (from 20.6 to 7.5).

An increase in the confidence in the diagnosis of neuropsychiatric syndromes was observed, along with trends of decrease in frequency for the diagnoses of psychotic syndrome (from $13.1 \%$ to $4.7 \%$ ), impulsive syndrome (from $14.0 \%$ to $7.5 \%$ ), maniform syndrome (from $4.7 \%$ to $0.0 \%$ ), and sleep alteration (from $15.9 \%$ to $8.4 \%$ ). Only the frequency of anxiety syndrome remained elevated (31.8\%) at the end of the study period (Table 3 and Figure 2).

\section{Discussion}

The external audit and the results herein reported show a complete elimination of physical and chemical restraints, along with an overall reduction of psychotropic medication, particularly for residents with dementia. In these patients, falls and emergency room referrals were also reduced. Overall, these results validate the CHROME criteria as a feasible, efficient, and safe tool to optimize the quality prescription of psychotropic medications in institutionalized people with dementia.

We believe that the definition of relatively stringent diagnostic criteria for people with dementia who present clinically significant neuropsychiatric syndromes (Appendix Table 2) turned out to be the primary key success factor to change prescription behavior, attain quality prescription, and secondarily reduce the frequency of the most dangerous medications. The observed trends of decrease in frequency of diagnoses for most neuropsychiatric syndromes suggests a previous overdiagnosis and supports the usefulness of the definitions provided. Figure 2 reflects how reduced frequency of diagnoses (all except anxiety syndrome) was associated with the reduction of neuroleptic, benzodiazepine, and hypnotic prescriptions.

The second key success factor had an organizational nature: attaining the Quality Seal of CHROME Criteria Compliance, which was awarded by an 
Table 3. Evolution of neuropsychiatric diagnoses $(n=107)$

\begin{tabular}{|c|c|c|c|c|c|}
\hline & $12 / 01 / 2015$ & $06 / 01 / 2016$ & $12 / 01 / 2016$ & $06 / 01 / 2017$ & $12 / 01 / 2017$ \\
\hline \multicolumn{6}{|c|}{ Depressive syndrome } \\
\hline Possible & NC & 6.5 & 5.6 & 5.6 & 0.9 \\
\hline Certain & NC & 8.4 & 6.5 & 8.4 & 12.1 \\
\hline Total & NC & $15.0(8.2-21.7)$ & $12.1(6.0-18.3)$ & $14.0(7.4-20.6)$ & $13.1(6.7-19.5)$ \\
\hline \multicolumn{6}{|c|}{ Anxiety syndrome } \\
\hline Possible & NC & 5.6 & 5.6 & 6.5 & 0.9 \\
\hline Certain & NC & 21.5 & 20.6 & 19.6 & 30.8 \\
\hline Total & NC & $27.1(18.7-35.5)$ & $26.2(17.8-34.5)$ & $26.2(17.8-34.5)$ & $31.8(23.0-40.6)$ \\
\hline \multicolumn{6}{|c|}{ Psychotic syndrome } \\
\hline Possible & NC & 2.8 & 1.9 & 0.9 & 0.0 \\
\hline Certain & $\mathrm{NC}$ & 10.3 & 11.2 & 10.3 & 4.7 \\
\hline Total & NC & $13.1(6.7-19.5)$ & $13.1(6.7-19.5)$ & $11.2(5.2-17.2)$ & $4.7(0.7-8.7)$ \\
\hline \multicolumn{6}{|c|}{ Impulsive syndrome } \\
\hline Possible & $\mathrm{NC}$ & 4.7 & 5.6 & 2.8 & 0.0 \\
\hline Certain & NC & 9.3 & 6.5 & 7.5 & 7.5 \\
\hline Total & NC & $14.0(7.4-20.6)$ & $12.1(6.0-18.3)$ & $10.3(4.5-16.0)$ & $7.5(2.5-12.5)$ \\
\hline \multicolumn{6}{|c|}{ Maniform syndrome } \\
\hline Possible & NC & 0.9 & 0.9 & 0.9 & 0.0 \\
\hline Certain & $\mathrm{NC}$ & 3.7 & 3.7 & 3.7 & 0.0 \\
\hline Total & NC & $4.7(0.7-8.7)$ & $2.8(0.7-8.7)$ & $4.7(0.7-8.7)$ & $0.0(0-0)$ \\
\hline \multicolumn{6}{|c|}{ Sleep alteration } \\
\hline Possible & NC & 4.7 & 2.8 & 5.6 & 0.0 \\
\hline Certain & NC & 11.2 & 10.3 & 8.4 & 8.4 \\
\hline Total & NC & $15.9(9.0-22.8)$ & $13.1(6.7-19.5)$ & $14.0(7.4-20.6)$ & $8.4(3.2-13.7)$ \\
\hline
\end{tabular}

Figures represent frequency (95\% confidence interval). NC: not collected.

external audit, motivated management, psychologists, physicians, and other staff to make the effort in treating behavioral or psychological symptoms with environmental and nonpharmacological strategies more thoughtfully. The temptation of using drugs as shortcuts was avoided through the stringency of the CHROME criteria.

In contrast to the rest of the syndromes, the prevalence of anxiety remained high at the end of the study. There are three explanatory factors for that result: 1) Sleep disorder or even impulsive and psychotic syndromes might have been reclassified to anxiety as the primary syndrome; 2) At the beginning of the study, physicians and psychologists did not clearly differentiate symptoms from syndromes. Put in a blunt example, sadness (reactive) due to nursing home placement does not necessarily meet depressive syndrome criteria; 3) Anxiety disorders are highly prevalent in older adults living in longterm care facilities (Seitz et al., 2010). In an observational study conducted in people with dementia, significant anxiety was found in $33.7 \%$ of the residents remaining so after one year $(31.2 \%)$ (Goyal et al., 2018).

The present study has several limitations. Though large, we described results of only one nursing home. Prevention of delirium and other potential physical benefits of the psychotropic medication reduction were not specifically recorded. The frequency and severity of behavioral and psychological symptoms at the beginning and end of the study were not quantitatively measured, as were not quality of life of residents and professional caregivers. The authors, nevertheless, believe that the changes in drug prescription did in no way worsen patient well-being (agitation, etc.). The four study psychologists (FC, MP, NR, AL) were vigilant in regard to the improvement-worsening of all residents and did not report any salient negative effects besides some residents being more demanding. Moreover, cases considered difficult were treated in clinical sessions with the study director (JO), and the principal investigator (RM) and solved acceptably.

The CHROME criteria require some investigative efforts on the physician's side, which might be perceived as more time consuming, especially in the beginning. Future research must clarify if optimized prescription settles in as a constant cultural component of the care home, since turnover of both residents and clinicians might thin a home's achievements over time.

Most articles published to improve prescription of psychotropics in people with dementia have focused on frequency (Janus et al., 2016; Richter et al., 2012), prescription factors (Brimelow et al., 


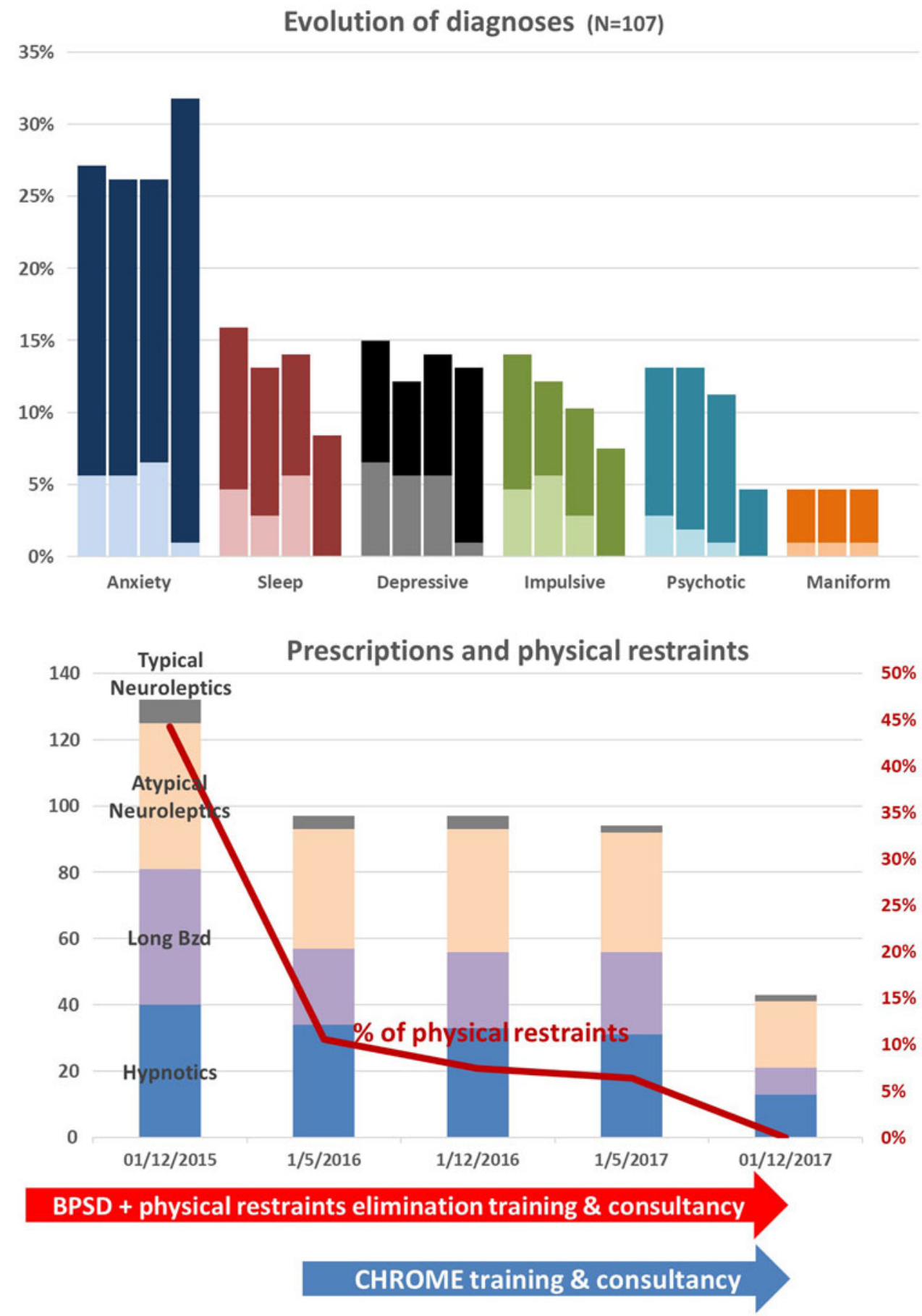

Figure 2. Evolution of certain (solid) and possible (light) neuropsychiatric diagnoses while physicians learned to use the CHROME criteria (up), total number of prescriptions for the most dangerous medications and frequency of physical restraints (down), across the study waves. BZD: benzodiazepines.

in press; Nørgaard et al., 2017), and BPSD. The "frequency and BPSD" approach has recently been described in an intervention using patient-centered medication reviews for BPSD (Massot Mesquida et al., 2019). In contrast to this more common approach, the CHROME criteria propose to focus on adequacy of prescription based on predefined neuropsychiatric syndromes, which appear as a result of a cerebral biological imbalance. From there, adequacy and frequency issues are implicitly solved in a relatively simpler and safer way (Appendix Tables 2 and 3).

The CHROME criteria can easily be applied by any physician, be it for community dwelling or 
institutionalized people with dementia. Clear definition of chemical restraint and ease for third-party verification add objectivity and organizational possibilities. The criteria can easily be adapted to other countries adjusting to local drugs, legal requirements concerning drug purchase, storage and disposal, as well as kinds of informed consent.

\section{Conflict of interest}

$\mathrm{RM}$ and JO received honoraria from ICOT for their consultancy and training services outside of this study.

\section{Description of the authors' roles}

R. Muñiz conceived the CHROME criteria, contributed to the study design, supervised the data collection, conducted statistical analyses, wrote part of the article, and coordinated all other coauthors' contributions. A.I. Pérez-Wehbe organized the study inception and reviewed the paper. F. Couto participated in neuropsychiatric diagnoses, supervised data extraction, and reviewed the paper. M. Pérez oriented physicians in treatment options, collected data, and reviewed the paper. N. Ramírez participated in neuropsychiatric diagnoses, collected data, and reviewed the paper. A. López supervised the entire study process, organized the external audits, and reviewed the paper. J. Rodríguez coordinated the physician's participation and reviewed the paper. T. Usieto and L. Lavin implemented prescriptions according to the CHROME criteria and gave valuable feedback to the research team. A. Rigueira provided CHROME criteria's pharmaceutical drug management and reviewed the manuscript. L. Agüera-Ortiz and J. LópezAlvarez contributed to the definition of neuropsychiatric syndromes and to the treatment guidelines and reviewed the paper. M. Martín-Carrasco assisted in writing the manuscript. J. Olazarán contributed to the definition of neuropsychiatric syndromes, participated in the study design, trained the study physicians, and wrote the paper.

\section{Acknowledgments}

The principal investigator and the study director thank Juan Luis Beltrán and Pilar García for their invaluable support regarding legal and bio-ethic issues related to both physical and pharmacological restraints that are woven into this article. Jesús $M^{a}$ López-Arrieta for his contributions from the geriatric perspective.

\section{Supplementary material}

To view supplementary material for this article, please visit https://doi.org/10.1017/S104161021900111X.

\section{References}

American Psychiatric Association. (2013). Diagnostic and Statistical Manual of Mental Disorders (DSM-5), 5th Ed. Arlington, VA: American Psychiatric Association.

Aranda-Gallardo, M. et al. (2015). [Consequences of errors in the translation of questionnaires: Spanish version of Downton index]. Revista de Calidad Asistencial, 30, 195202. doi: 10.1016/j.cali.2015.04.003. [Article in Spanish]

Ballard, C. et al. (2009). The dementia antipsychotic withdrawal trial (DART-AD): long-term follow-up of a randomised placebo-controlled trial. Lancet Neurology, 8, 151-157. doi: 10.1016/S1474-4422(08)70295-3.

Brimelow, R. E., Wollin, J. A., Byrne, G. J. and Dissanayaka, N. N. (in press). Prescribing of psychotropic drugs and indicators for use in residential aged care and residents with dementia. International Psychogeriatrics, 31, 837-847. doi: 10.1017/S1041610218001229.

Cid-Ruzafa, J. and Damián-Moreno, J. (1997). [Disability evaluation: Barthel's index]. Revista Española de Salud Pública, 71, 127-137. [Article in Spanish]

Goyal, A. R., Bergh, S., Engedal, K., Kirkevold, M. and Kirkevold, Ø. (2018). The course of anxiety in persons with dementia in Norwegian nursing homes: a 12-month follow-up study. Fournal of Affective Disorders, 235, 117-123. doi: 10.1016/j.jad.2018.04.015.

Hughes, C. M. and Lapane, K. L. (2005). Administrative initiatives for reducing inappropriate prescribing of psychotropic drugs in nursing homes: how successful have they been? Drugs \& Aging, 22, 339-351.

Janus, S. I., van Manen, J. G., IJzerman, M. J. and Zuidema, S. U. (2016). Psychotropic drug prescriptions in Western European nursing homes. International Psychogeriatrics, 28, 1775-1790.

Ljunggren, G., Phillips, C. and Sgadari, A. (1997). Comparisons of restraint use in nursing homes in eight countries. Age E Ageing, 26(Suppl 2), 43-47.

Lobo, A. et al. (1999). [Revalidation and standardization of the cognition mini-exam (first Spanish version of the MiniMental Status Examination) in the general geriatric population]. Medicina Clínica (Barcelona), 112, 767-774. [Article in Spanish]

Majic, T. et al. (2010). The pharmacotherapy of neuropsychiatric symptoms of dementia: a cross-sectional study in 18 homes for the elderly in Berlin. Deutsches Ärzteblatt International, 107, 320-327. doi: 10.3238/arztebl. 2010.0320.

Massot Mesquida, M., Tristany Casas, M., Franzi Sisó, A., García Muñoz, I., Hernández Vian, Ó. and Torán Monserrat, P. (2019). Consensus and evidence-based medication review to optimize and potentially reduce psychotropic drug prescription in institutionalized dementia patients. BMC Geriatrics, 19, 7. doi:10.1186/ s12877-018-1015-9.

Muñiz, R. et al. (2016). Reducing physical restraints in nursing homes: A report from Maria Wolff and Sanitas. 
Fournal of the American Medical Directors Association, 17, 633-639. doi: 10.1016/j.jamda.2016.03.011.

Nørgaard, A., Jensen-Dahm, C., Gasse, C., Hansen, E. S. and Waldemar, G. (2017). Psychotropic polypharmacy in patients with dementia: prevalence and predictors. fournal of Alzheimer's Disease, 56, 707-716. doi: 10.3233/ JAD-160828.

Olazarán, J., Valle, D., Serra, J. A., Cano, P. and Muñiz, R. (2013). Psychotropic medications and falls in nursing homes: a cross-sectional study. Fournal of the American Medical Directors Association, 14, 213-217. doi: 10.1016/j. jamda.2012.10.020.

Olazarán-Rodríguez, J. et al. (2016). [The CHROME criteria for the accreditation of centers free of chemical restraints and for a quality prescription of psychotropic medications]. Psicogeriatría, 6, 91-98. [Article in Spanish]

Phillips, C. D., Spry, K. M., Sloane, P. D. and Hawes, C. (2000). Use of physical restraints and psychotropic medications in Alzheimer special care units in nursing homes. American fournal of Public Health, 90, 92-96.

Richter, T., Mann, E., Meyer, G., Haastert, B. and Köpke, S. (2012). Prevalence of psychotropic medication use among German and Austrian nursing home residents: a comparison of three cohorts. Fournal of the American Medical Directors Association, 13, 187.e7-187.e13. doi: 10.1016/j.jamda.2011.03.007.

Schneider, L. S., Dagerman, K. S. and Insel, P. (2005). Risk of death with atypical antipsychotic drug treatment for dementia: meta-analysis of randomized placebocontrolled trials. Fournal of the American Medical Association, 294, 1934-1943.

Seitz, D., Purandare, N. and Conn, D. (2010). Prevalence of psychiatric disorders among older adults in long-term care homes: a systematic review. International Psychogeriatrics, 22, 1025-1039. doi: 10.1017/S1041610210000608. 